\title{
Isoprostanes as physiological mediators of transition to newborn life: novel mechanisms regulating patency of the term and preterm ductus arteriosus
}

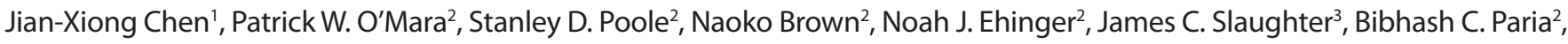 \\ Judy L. Aschner' ${ }^{2}$, Jeff Reese ${ }^{2,4}$
}

BACKGROUND: Increased oxygen tension at birth regulates physiologic events that are essential to postnatal survival, but the accompanying oxidative stress may also generate isoprostanes. We hypothesized that isoprostanes regulate ductus arteriosus (DA) function during postnatal vascular transition.

METHODS: Isoprostanes were measured by gas chromatographymass spectrometry. DA tone was assessed by pressure myography. Gene expression was measured by quantitative PCR.

RESULTS: Oxygen exposure was associated with increased 8-iso-prostaglandin (PG)F2a in newborn mouse lungs. Both 8-iso-PGE2 and 8-iso-PGF2ainduced concentration-dependent constriction of the isolated term DA, which was reversed by the thromboxane A2 (TXA2) receptor antagonist SQ29548. SQ29548 pretreatment unmasked an isoprostane-induced DA dilation mediated by the EP4 PG receptor. Exposure of the preterm DA to 8-iso-PGE2 caused unexpected DA relaxation that was reversed by EP4 antagonism. In contrast, exposure to 8-iso-PGF2a caused preterm DA constriction via TXA2 receptor activation. Further investigation revealed the predominance of the TXA2 receptor at term, whereas the EP4 receptor was expressed and functionally active from mid-gestation onward. CONCLUSION: This study identifies a novel physiological role for isoprostanes during postnatal vascular transition and provide evidence that oxidative stress may act on membrane lipids to produce vasoactive mediators that stimulate physiological DA closure at birth or induce pathological patency of the preterm DA.

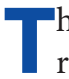
he ductus arteriosus (DA) is a central vascular shunt that remains widely patent during fetal life but rapidly constricts soon after birth, allowing redirection of blood flow from the fetal gas exchange organ, the placenta, to the newly inflated lungs. Postnatal DA constriction is mediated, in part, by increased oxygen tension, withdrawal of vasodilatory prostaglandins (PGs), and stimulation of ion channels. Unfortunately, the DA fails to close in a large proportion of preterm infants, resulting in persistent patency of the DA (PDA), one of the most common congenital cardiac disorders (1).

Oxidative stress is a feature of numerous pathological conditions that occur in the perinatal period (2-4). Newborns are subjected to oxidative stress as a result of rapid transition from a low-oxygen environment in utero to a relatively high-oxygen environment at birth (5). F2-isoprostanes, an established marker for oxidative stress (6), are PGF2-like compounds produced nonenzymatically by free radical-mediated peroxidation of arachidonic acid. Increased levels of plasma F2-isoprostanes have been demonstrated in newborns as compared with healthy adults $(7)$ and in infants under duress $(8,9)$. Although 8-iso-PGE2 and 8-iso-PGF2 $\alpha$ mediate vasoconstriction in different vascular beds $(10,11)$, there is little information on isoprostanes as potential mediators of postnatal DA closure $(12,13)$.

Recent reports demonstrate that 8-iso-PGF2 $a$ contributes to pulmonary hypertension in the newborn rat (14). In addition, 8-iso-PGF2 $\alpha$ levels were increased following 7-14 d of exposure to $60 \%$ oxygen in neonatal rats, whereas administration of a lipid hydroperoxide scavenger suppressed lung isoprostane levels and prevented right ventricular hypertrophy (15). On the basis of these reports, we hypothesized that isoprostane levels would be increased shortly after birth due to the abrupt shift in postnatal oxygen tension, and that isoprostanes would contribute to postnatal DA constriction.

\section{RESULTS}

Brief Oxygen Exposure at Birth Stimulates Isoprostane Formation

We measured 8-iso-PGF2 $\alpha$ levels in lung tissue from fetal and newborn mice using gas chromatography/negative-ion chemical ionization mass spectrometry. These data show that 8-iso-PGF2 $\alpha$ was detectable in the lungs of term gestation fetuses under in utero oxygen conditions. There was a trend toward increased 8 -iso-PGF2 $\alpha$ levels after $4 \mathrm{~h}$ when pups were 
delivered into room air. Littermates exposed to $80 \%$ oxygen had a significant increase in pulmonary 8-iso-PGF2a levels (Figure 1), similar to reports of elevated 8-iso-PGF2 $\alpha$ levels following 4-7 d of hyperoxia (16). This was accompanied by rapid closure of the DA (Figure 2a,b), suggesting a temporal association between generation of isoprostanes or other reactive oxygen species (ROS) under increased oxygen conditions soon after birth and during closure of the DA at term gestation.

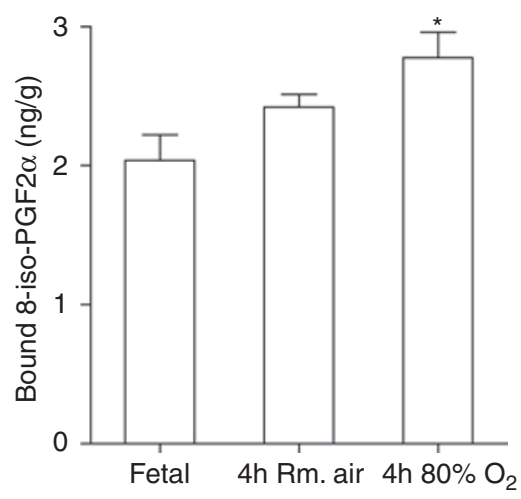

Figure 1. Generation of isoprostanes in the newborn mouse lung. Bound levels of 8-iso-PGF2a in fetal and newborn lungs were determined by gas chromatography-mass spectrometry. Term gestation (d19) offspring were delivered by caesarean section and divided into three groups. Pups were either killed immediately ("fetal") or at $4 \mathrm{~h}$ of life after exposure to room air (" $4 \mathrm{~h} \mathrm{Rm}$. air") or $80 \% \mathrm{O}_{2}$ for the entire $4 \mathrm{~h}$ (" $4 \mathrm{~h} 80 \% \mathrm{O}_{2}$ ") ( $n=8$ pups/group from four litters). Data are mean \pm SEM. $* P<0.01$ as compared with fetal lungs.
Isoprostanes Constrict the Term DA via the Thromboxane Receptor

The direct vascular responses of isolated term and preterm murine DAs to 8-iso-PGF2 $\alpha$ and 8-iso-PGE2 were examined under different oxygen conditions. Exposure to 8-iso-PGF2a produced concentration-dependent vasoconstriction under fetal oxygen conditions (Figure 2c,e). More significant constriction occurred under conditions that mimic newborn oxygen tension (Figure 2e). Similarly, exposure of the isolated DA to 8 -iso-PGE2 resulted in concentration-dependent vasoconstriction (Figure 2d).

To examine the potential mechanisms by which isoprostanes induce DA constriction, isolated term DAs were exposed to 8 -iso-PGE2 or 8-iso-PGF2 a followed by treatment with a thromboxane A2 (TxA2) receptor antagonist, because isoprostanes can bind and activate various eicosanoid receptors $(17,18)$. Exposure to either isoprostane species produced intense vasoconstriction and near-complete closure of the DA lumen (Figure 2d-g). These effects were completely reversed by subsequent addition of the TxA2 receptor antagonist, SQ29548 (Figure 2f-g), indicating that isoprostane-induced DA constriction is primarily mediated via activation of the TxA2 receptor.

\section{Thromboxane Receptor Blockade Reveals Isoprostane-Induced DA Dilation}

Contrary to expectation, exposure to increasing concentrations of 8-iso-PGE2 produced potent relaxation of the isolated DA when ductus TxA2 receptors were first blocked by pretreatment a

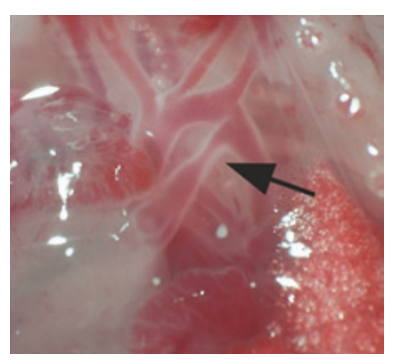

b
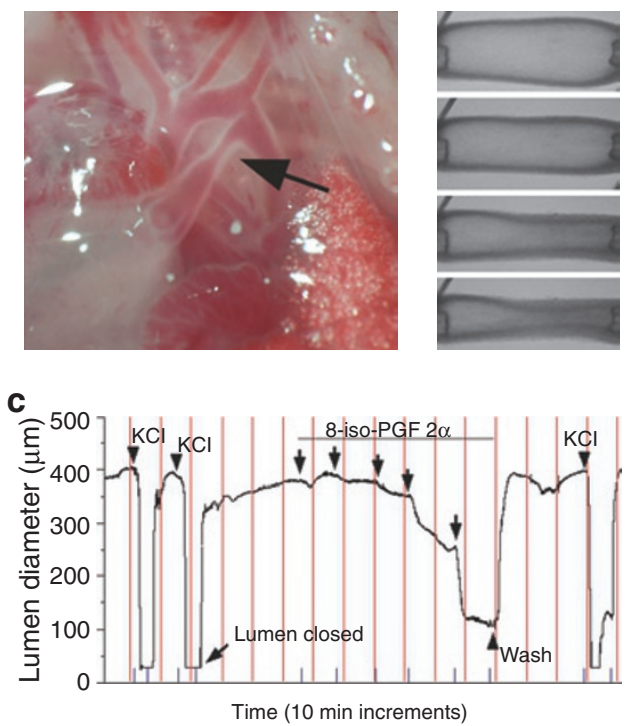

d

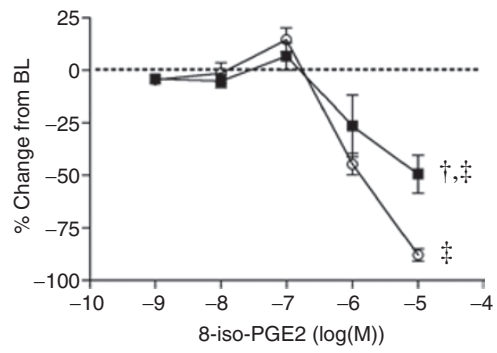

e

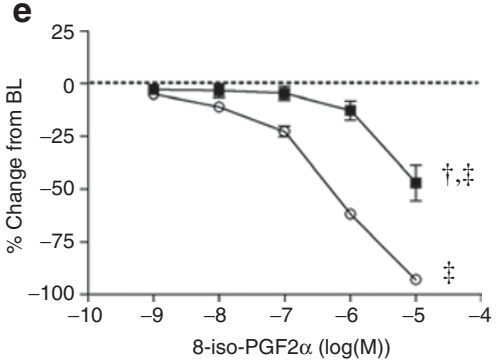

f
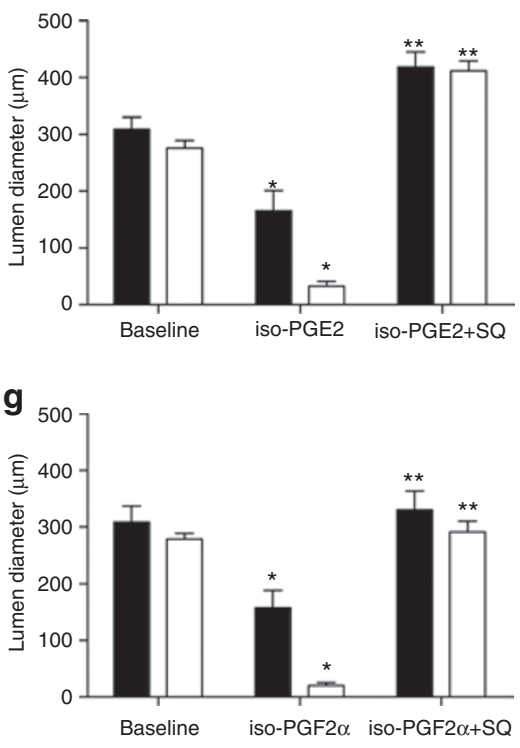

Figure 2. Responses of the term (d19) ductus arteriosus (DA) to isoprostanes under fetal and newborn oxygen conditions. Oxygen exposure constricts the DA in vivo (a) (1 h, room air; arrow indicates DA) and in vitro (b) (5-min time-lapse windows; $21 \%$ oxygen). Changes in vasomotor tone were examined by cannulated, pressurized vessel myography under continuous video microscopy. Exposure to 8-iso-prostaglandin (PG)F2a elicited a concentration-dependent constriction of the isolated DA (c). 8-Iso-PGE2-induced DA constriction from baseline ("BL") was more potent under newborn (white circles; $n=7$ ) than fetal (black squares; $n=8$ ) oxygen conditions (d); this difference was more evident in response to 8-iso-PGF2a (newborn (white circles; $n=7$ ); fetal (black squares; $n=11)(\mathbf{e})$. These effects were reversed by the subsequent addition of the thromboxane receptor antagonist, SQ29548 ("SQ") (white bars = newborn oxygen conditions $(n=7)$; black bars = fetal oxygen conditions $(n=3))(\mathbf{f}, \mathbf{g})$. Data are mean \pm SEM. $+P<0.01$, fetal vs. newborn oxygen conditions. $¥ P<0.01$ for concentration-dependent change in lumen diameter; ${ }^{*} P<0.01$ as compared with baseline; ${ }^{* *} P<0.01$ as compared with isoprostane-induced constriction. 
with SQ29548 (Figure 3a). 8-Iso-PGF2a induced a similar vasodilatory response after TxA2 antagonism, albeit at higher concentrations (Figure $3 b$ ).

To better understand the vasodilatory response of the DA to isoprostanes, the term DA was pretreated with the nitric oxide synthase inhibitor, N-(G)-nitro-L-arginine methyl ester (L-NAME), the cyclooxygenase inhibitor indomethacin, or the EP4-specific receptor antagonist L-161982 before treatment with SQ29548 and exposure to 8-iso-PGE2 or 8-iso-PGF2a. Pretreatment of the term DA with L-161982 caused significant reduction in the vasodilatory response to increasing concentrations of 8 -iso-PGE2 $\left(10^{-9}-10^{-5} \mathrm{~mol} / \mathrm{l}\right)$ after TxA2 receptor blockade (Figure 3a). Pretreatment with L-NAME or indomethacin had no effect on the concentration-dependent vasodilatory response to 8 -iso-PGE2 (Figure 3a). 8-Iso-PGF2a
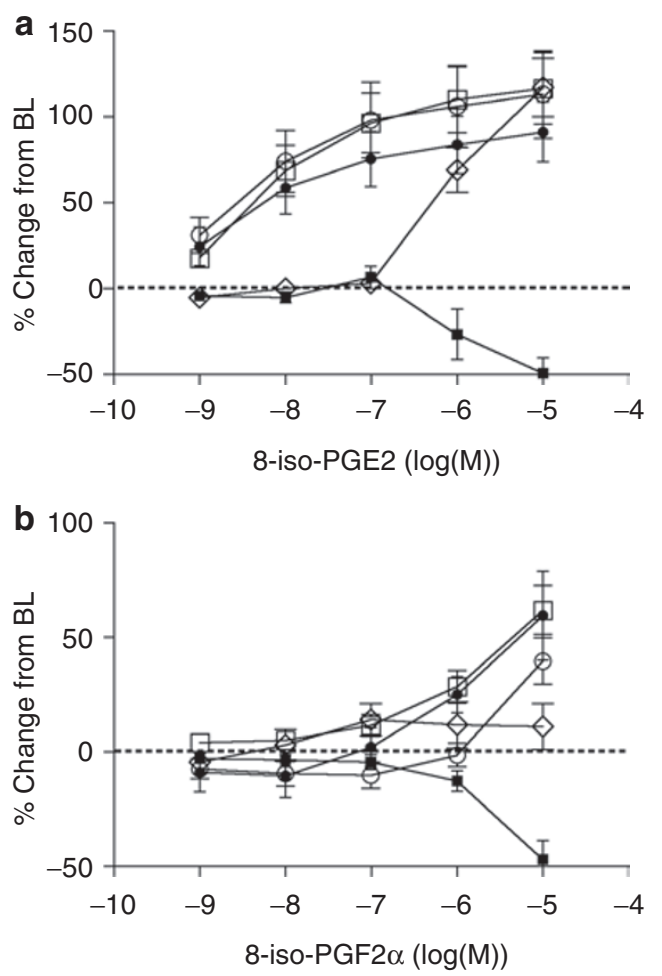

Figure 3. Isoprostane-induced vasodilation of the ductus arteriosus (DA) after thromboxane A2 (TxA2) receptor antagonism. (a) Exposure to 8-isoprostaglandin (PG)E2 caused constriction of the isolated DA (black squares; $n=8$ ) (values from Figure 2) whereas pretreatment with the TxA2 receptor antagonist, SQ29548 $\left(10^{-5} \mathrm{~mol} / \mathrm{I}\right)$, revealed a potent vasodilatory response to increasing doses of 8-iso-PGE2 (white squares; $n=4)(P<0.01)$. 8-IsoPGE2-induced vasodilation was not affected by preincubation with inhibitors of nitric oxide $\left(10^{-4} \mathrm{~mol} / \mathrm{l} \mathrm{N}-(\mathrm{G})\right.$-nitro-L-arginine methyl ester (L-NAME)) (black circles; $n=5)(P=0.99)$ or PG synthesis $\left(10^{-5} \mathrm{~mol} / \mathrm{l}\right.$ indomethacin, "Indo") (white circles; $n=4)(P=0.95)$, but was significantly inhibited by pretreatment with the EP4 antagonist, L-161982 (white diamonds; $n=4$ ) ( $P=0.02$, as compared with SQ29548 pretreatment alone). (b) Although exposure of the isolated DA to 8-iso-PGF2a also caused DA constriction (black squares; $n=11$ ) (values from Figure 2), pretreatment with SQ29548 induced a vasodilatory response only at higher 8-iso-PGF2a concentrations $(P<0.01)$. Preincubation with L-NAME (black circles; $n=6)(P=0.55)$ or indomethacin (white circles; $n=6)(P=0.08$ ) had little or no effect on DA dilation, but pretreatment with the EP4 antagonist, L-161982, blocked the vasodilatory response to 8 -iso-PGF2a (white diamonds; $n=7)(P<0.01$, as compared with SQ29548 pretreatment alone). Data are mean \pm SEM. likewise induced DA dilation following TxA2 receptor blockade (Figure $3 \mathbf{b}$ ) that was independent of nitric oxide (NO) and PG synthesis, and mediated via EP4 signaling. These data suggest that the underlying mechanism for 8-iso-PGE2- and 8 -iso-PGF2 $\alpha$-induced dilation of the term DA is activation of the EP4 receptor by isoprostanes, and not synthesis of NO PGE2 or other PGs in the ductus wall.

\section{Isoprostane-Mediated Effects on DA Tone Are Developmentally Regulated}

To explore the maturational dependency of TxA2 and EP4 receptor regulation of isoprostane-induced $\mathrm{DA}$ responses, we examined the effects of 8 -iso-PGE2 and 8-iso-PGF2 $\alpha$ in the preterm DA. In contrast to term vessels, exposure of the preterm (day 15 of gestation; d15) DA to 8-iso-PGE2 led to an unexpected dose-dependent vessel relaxation (Figure $4 \mathbf{a}, \mathbf{b}$ ); this effect could be reversed by EP4 antagonism (Figure 4c). Pre-incubation of the preterm DA with the EP4 antagonist L-161982 completely blocked 8-iso-PGE2-induced vessel relaxation (Figure 4d). By comparison, exposure of the preterm DA (d15) to 8-iso-PGF2 $\alpha$ resulted in dose-dependent vessel constriction (Figure $4 \mathrm{e}, \mathrm{f}$ ) that was mediated via the TxA2 receptor (Figure 4g), similar to responses in term vessels. 8-IsoPGF2 $\alpha$-induced vessel constriction was completely blocked by pretreatment with the TxA2 receptor antagonist SQ29548 (Figure 4h).

\section{Gestation-Specific Isoprostane Effects Are Determined by Shift in Target Receptors}

One possible explanation for the differential response of term and preterm vessels to different isoprostane moieties is a gestation-specific shift in the expression of their target receptors. During mid-to-late gestation, TxA2 receptor expression remained low in the DA until the day of parturition (day 19 of pregnancy; d19). Correspondingly, the synthetic Tx ana$\log$ U-46619 had limited contractile effects in the preterm DA but highly potent vasoconstrictive effects at term gestation (Figure 5b). In contrast, expression of EP4 was upregulated early and was sustained until after birth (Figure 5a,c). Notably, the preterm DA was highly sensitive to EP4-mediated dilation, similar to the term DA (Figure 5d). These results indicate that opposing, gestation-specific isoprostane effects on the term vs. preterm DA are determined by maturational changes in TxA2 and EP4 receptors.

\section{DISCUSSION}

In this study, we show that isoprostane levels are increased shortly after birth in response to increased oxygen tension and that isoprostane generation may serve as a novel physiological signal to stimulate postnatal DA closure. We unexpectedly found that isoprostanes have both vasoconstrictive and vasodilatory effects, depending on gestational maturity, oxygen tension, and developmental expression of target receptors. These findings support a potential role for isoprostanes in the vascular transition process after birth. Although animal studies should be interpreted with caution with respect to any human 

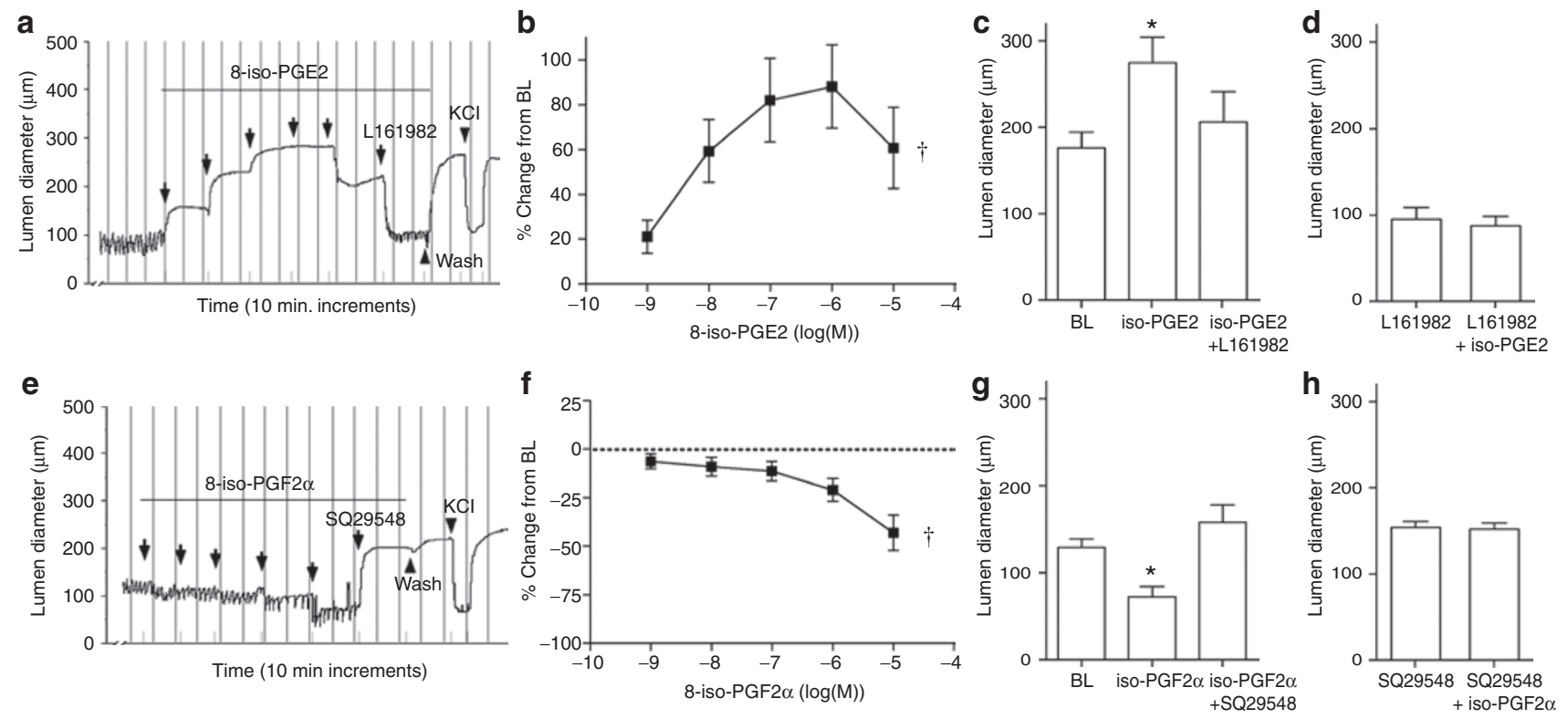

Figure 4. Opposing effects of isoprostanes in the preterm (d15) ductus arteriosus (DA). (a, b) Exposure to 8-iso-prostaglandin (PG)E2 caused concentration-dependent dilation of the isolated preterm DA, although a modest contractile response was noted at the highest dose $(n=6)$. (c) 8-iso-PGE2-induced vasodilation $\left(10^{-5} \mathrm{~mol} / \mathrm{l}\right)$ was reversed by addition of the EP4 antagonist, L-161982 $\left(10^{-6} \mathrm{~mol} / \mathrm{l} ; n=6\right)$. (d) L-161982 also prevented vasodilatory effects induced by $10^{-5} \mathrm{~mol} / / \mathrm{l}$ 8-iso-PGE2 $(n=5)$. (e, f) In contrast, 8-iso-PGF2a elicited DA constriction $(n=5)$. (e, g) This was reversed by the addition of $10^{-6} \mathrm{~mol} / \mathrm{I} \mathrm{SQ} 29548(n=5)$. (h) Preincubation with SQ29548 blocked the vasoconstrictive effects induced by $10^{-5} \mathrm{~mol} / \mathrm{l}$ 8-iso-PGF2a $(n=5)$. Data are mean \pm SEM. $+P<0.01$ for concentration-dependent change in lumen diameter; ${ }^{*} P<0.05$ as compared with baseline (BL).

implications, these studies suggest that premature infants may be at risk for prolonged PDA with exposure to increased oxygen tension and signaling via vasodilatory EP4 receptors.

Membrane lipids are susceptible to peroxidation at sites of free radical generation, resulting in formation of isoprostanes. Isoprostanes are subsequently released by the action of phospholipases and are stable products in circulation. Increased isoprostane levels in plasma and other body fluids are indicative of oxidative stress and are commonly used as biomarkers of injury in the cardiovascular system and various other cells and tissues (6). However, circulating isoprostanes can also act as intracellular signaling molecules and typically exert vasoconstrictive effects in most vascular beds, as well as smooth muscle constriction of the lymphatic, uterine, gastrointestinal, and tracheobronchial systems (19). The vascular effects of isoprostanes also include platelet activation and adhesion of neutrophils and monocytes to the endothelium $(20,21)$. Recent studies show that monocyte adhesion (22) and platelet aggregation (23) contribute to postnatal DA constriction, suggesting a potential role for isoprostanes in the DA closure process. We speculated that the vasoconstrictive and vascular occlusive properties of isoprostanes might contribute to DA closure after birth. Our data show that 8-iso-PGF2a, one of the most frequently used markers of oxidative stress, is increased in newborn lungs exposed to a short period of increased oxygen tension. Although isoprostanes are generally not recognized as mediators of DA function, we observed potent vasoconstrictive effects on the isolated DA at term gestation. These results confirm a recent study in the chick ductus (13). Isoprostanes are associated with pathological conditions in nearly every biological system and are typically considered to have detrimental effects. However, these data suggest that isoprostanes may be a molecular indicator of the acute shift in oxygenation at birth and perform a physiological role by stimulating circulatory adaptation to an oxygen-enriched environment.

Newborn infants experience oxidative stress as part of the normal process of labor and delivery (24). Interestingly, the F2-isoprostanes generated at birth in healthy, breast-feeding term infants do not return to adult levels until 6 mo of age (25), suggesting a slow adaptive process to oxidative stress in infancy. Oxidative stress is also increased during premature birth $(3,5,7)$ or complicated pregnancies $(2,4,25,26)$ and in various neonatal pathologies, including retinopathy of prematurity, bronchopulmonary dysplasia, hypoxic-ischemic encephalopathy, periventricular leukomalacia, intrauterine growth restriction, and hemolysis $(2,3,27)$. Recent studies also show that delayed closure of the DA after birth leaves the newborn at risk for disorders that are associated with oxidative stress (12), and that premature infants with PDA have increased isoprostane levels that decline with treatment (28). Most of these studies were correlative and based on associations with increased levels of plasma or urine 8-iso-PGF2 $\alpha$. We used an isolated vessel preparation to directly demonstrate that 8 -iso-PGF $2 \alpha$ and 8-iso-PGE2 cause intense constriction of the mature DA under fetal and neonatal oxygen conditions. Although an in vitro approach was required to address this hypothesis, the doses used for these studies correspond to the levels of isoprostanes found in term and preterm infants $(7,25,26)$ and are similar to those used in neonatal vascular studies (12). Moreover, isoprostanes are generated at the site of oxidant injury and may be 
a
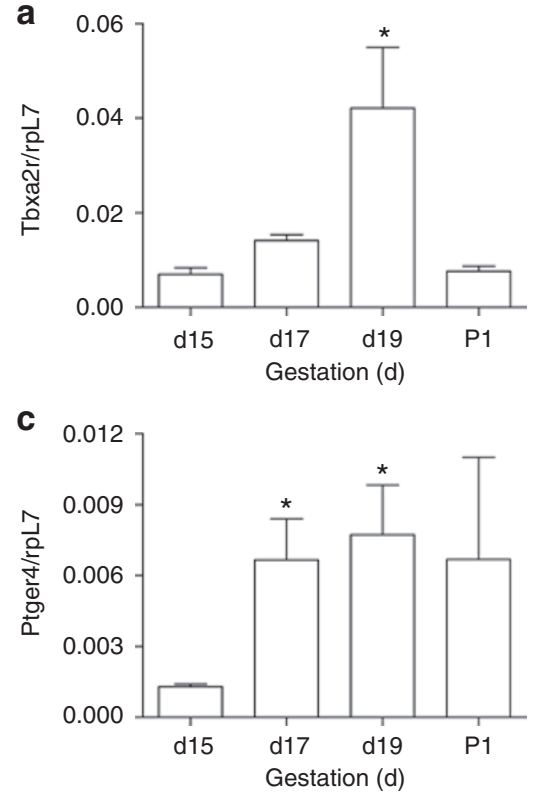
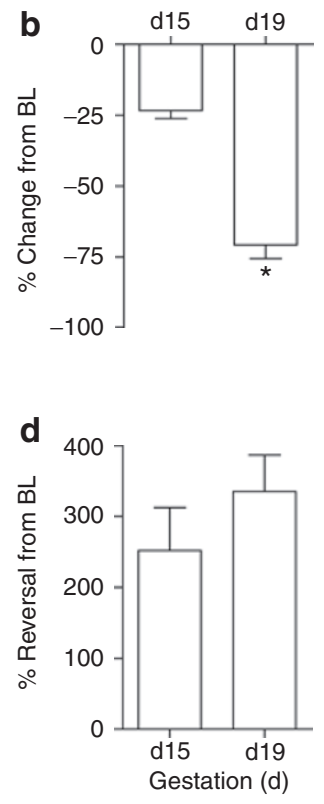

Figure 5. Maturation-specific changes in isoprostane target receptors. The expression and function of receptors commonly used by isoprostanes were analyzed. (a) The thromboxane receptor, Tbxa2r, was upregulated in the term (d19) ductus arteriosus (DA) by quantitative PCR and (b) was highly sensitive to stimulation by the synthetic agonist, U-46619 $\left(10^{-8} \mathrm{~mol} / \mathrm{l}\right)$, at term, but not preterm, gestation. (c) In contrast, Ptger4, encoding the EP4 receptor for prostaglandin (PG)E2, was upregulated at an earlier time point and remained elevated. (d) Stimulation of EP4 by PGE2 $\left(10^{-6} \mathrm{~mol} / \mathrm{l}\right)$ caused a similar degree of vasodilation of both the preterm and term DA. ${ }^{*} P<0.05$ as compared with $\mathrm{d} 15$.

diluted in the systemic circulation, suggesting that local concentrations in the DA or the pulmonary system may be higher and exert more potent regional vascular effects (10). Other investigators have demonstrated that ROS induce constriction of the DA via mitochondrial redox-sensing mechanisms $(29,30)$. Subsequent stimulation of smooth muscle contraction by ROS-mediated inhibition of specific voltage-gated potassium channels and activation of Rho-kinase signaling helps to initiate DA constriction (30-32). These data support the current concept that products of oxidative stress, in this case, the generation of isoprostanes, may be one of the initial events triggering DA closure after birth.

The vasoconstrictor effects of isoprostanes are typically transduced by the TxA2 receptor, although isoprostanes also serve as ligands for other PG receptors, including the PGE receptors EP1-4 $(17,18,33)$, as well as the peroxisome proliferator activated receptors (34). Previous studies show that the cyclooxygenase-PGE2-EP4 receptor signaling pathway plays a critical role in regulation of DA maturation and closure after birth (35-39). EP4 and the TxA2 receptor TP are the predominant PG receptors in the DA of mice, humans, and other species $(37,40,41)$, in which their effects are developmental stagespecific. Our data show that 8-iso-PGE2 and 8-iso-PGF2a have both contractile and vasodilatory effects on the DA depending on relative predominance of the TP and EP4 receptors. With increasing maturity, the balance between EP4 and

TP shifted in favor of the contractile effects of TP stimulation at term gestation. These findings concur with Loftin et al. (36), who showed that TP agonists could forcefully close the PDA of cyclooxygenase-deficient mice at term gestation. Their subsequent studies demonstrated high levels of TP expression in the developing fetal mouse DA, although no functional studies were performed to determine the significance of TP expression at each gestation (42). Using an ex vivo approach, we found that blockade of the TP receptor unmasked the vasodilatory effects of 8-iso-PGE2 and 8-iso-PGF2 $\alpha$ in the term DA. By comparison, 8-iso-PGE2 caused unexpected vasodilation of the preterm DA without prior TP antagonism. Our antagonist studies show that relaxation of the term and preterm DA by isoprostanes can be attributed to EP4 receptor stimulation. These results may have clinical relevance because EP4 is the most important PGE receptor in preterm mice and humans and PDA is a critical dilemma for many premature infants in the neonatal intensive care unit. The development of selective EP4 receptor antagonists for therapeutic use in preterm infants with symptomatic PDA might block the vasodilatory effects of endogenous PGs as well as the stimulatory effects of 8-iso-PGE2.

Both E- and F-ring isoprostanes can stimulate vasodilation under certain circumstances. Previous studies show that 8-isoPGF2 $\alpha$ has a dual effect on pulmonary arteries of adult rats $(14,43)$. In those tissues, 8 -iso-PGF2 $\alpha$ induced a contractile response in relaxed pulmonary arteries, whereas 8-iso-PGF2 $\alpha$ caused relaxation in the pre-contracted pulmonary artery via a mechanism involving activation of the nitric oxide synthase-NO pathway $(14,43)$. In contrast to newborn pulmonary arteries, our results indicate that isoprostane-mediated relaxation of the DA is independent of NO production. Relaxation of the DA in utero is an active process that is primarily regulated by NO synthesis in preterm fetuses and by vasodilatory PGs in the term fetal DA $(42,44)$. Our study identifies isoprostanes as another mechanism for relaxation of the premature DA that is independent of both NO and PG synthesis. Thus, isoprostanemediated vasodilation of the immature DA may be an entirely novel explanation for the failure of DA closure in premature infants. Moreover, clinical tolerance or promotion of increased oxygen levels in order to stimulate DA closure in preterm neonates might be called into question by these results.

Overall, these data reveal a previously unrecognized role for isoprostanes or their metabolites in DA regulation, using signaling pathways that are influenced by oxygen tension and gestational age. Formation of ROS can occur under physiological conditions and is essential for certain cell signaling processes (45). Many types of ROS are generated in response to hyperoxia, which might contribute to DA constriction. However, lipid peroxidation leading to formation of isoprostanes is considered deleterious in most biological systems. Our results suggest that exposure to increased oxygen levels at the time of birth may be one context in which isoprostane formation serves a physiological role. These findings are relevant to our understanding of the pathogenesis of preterm PDA and the potential link between oxidative stress and circulatory adaptation at birth. 


\section{METHODS}

\section{Animals and Tissues}

Experiments were conducted in accordance with National Institutes of Health animal care standards and were approved by the Institutional Animal Care and Use Committee at Vanderbilt University. Adult female CD1 mice (Charles River, Raleigh, NC) were bred to produce timed pregnancies (copulatory plug $=$ day 1 ). For myography studies, pregnant females were anesthetized by intraperitoneal injection of $0.4 \mathrm{ml} 2.5 \%$ avertin (2,2,2-tribromoethanol in tert-amyl alcohol; Sigma-Aldrich, St Louis, MO), followed by isoflurane inhalation (Baxter, Deerfield, IL) to facilitate fetal anesthesia. For lung tissue collection, dams were euthanized by cervical dislocation at term gestation (day 19 of pregnancy) and offspring were delivered by uterine incision. Term newborn pups were exposed to either room air or $80 \%$ oxygen for $4 \mathrm{~h}$, then briefly anesthetized with isoflurane and euthanized by cervical transection. Lung tissues from fetuses (day 19, 09:00) and their littermates (reared in different oxygen conditions until $4 \mathrm{~h}$ of age) were collected immediately after killing and snap frozen for later analysis. For gene expression studies, whole DA segments were obtained from fetuses at 09:00 on gestation days 15, 17, and 19 and from postnatal day 1 pups (09:00). Ductus segments were cleanly excised from surrounding tissues and promptly homogenized in RNA buffer (RNeasy; Qiagen, Valencia, CA). Total RNA was extracted, treated with DNaseI according to the manufacturer's recommendations, and stored at $-80^{\circ} \mathrm{C}$ for later analysis.

\section{Myography Studies}

Anesthetized fetuses were submerged in ice-cold, deoxygenated (95\% $\mathrm{N}_{2}, 5 \% \mathrm{CO}_{2}$ ) Krebs buffer and secured. Krebs buffer was modified (in mM: $109 \mathrm{NaCl}, 34 \mathrm{NaHCO}_{3}, 4.7 \mathrm{KCl}, 0.9 \mathrm{MgSO}_{4}, 1.0 \mathrm{KH}_{2} \mathrm{PO}_{4}, 11.1$ dextrose, and $\left.2.5 \mathrm{CaCl}_{2}\right)$ to maintain stable $\mathrm{pH}(7.30-7.35)$ and relative hypoxia in the vessel bath (dissolved oxygen content $=1.5-1.8 \%$; measured $\mathrm{pO}_{2}=38-45$ Torr). Isolation of the fetal ductus was performed as described previously $(38,39)$. Isolated DAs were transferred to custom microvessel perfusion chambers (Instrumentation and Model Facility, The University of Vermont, Burlington, VT) filled with chilled, deoxygenated Krebs buffer. The excised vessel preparation was positioned and secured on $\sim 120$ - $\mu$ m pipette tips, as previously described (39).

Once mounted, perfusion chambers were placed on an inverted microscope equipped with a video camera and image-capture system (IonOptix, Milton, MA) to continuously record the intraluminal diameter. Optical markers used to detect the lumen diameter were positioned at the narrowest point of the constricted vessel. Vessels were allowed to equilibrate at $37^{\circ} \mathrm{C}$ for $30-40 \mathrm{~min}$ under deoxygenated conditions, then pressurized in a stepwise manner to $20 \mathrm{~mm} \mathrm{Hg}$ (term) or $6 \mathrm{~mm} \mathrm{Hg}$ (preterm), in accordance with previous studies (39). After equilibration, vessels were exposed to $50 \mathrm{mM} \mathrm{KCl}(\times 2)$ to ascertain vessel reactivity. In some experiments, the Krebs buffer was bubbled with $12.5 \%$ oxygen to simulate newborn oxygen conditions (39). Subsequent studies were performed with 8-iso-PGE2 and 8-iso-PGF2 $\alpha$ (or 15-E2t-IsoP and 15-F2t-IsoP, respectively, the most common E- and F-ring isoprostanes) $\left(10^{-9}-10^{-5} \mathrm{~mol} / \mathrm{l}\right.$; Cayman Chemical, Ann Arbor, MI). Drugs and doses used for the myography experiments (39) included: the TxA2 receptor agonist U-46619 $\left(10^{-8}\right.$ mol/l; Cayman Chemical), the TxA2 receptor antagonist SQ29548 $\left(10^{-6} \mathrm{~mol} / \mathrm{l}\right.$; Cayman Chemical), the nonselective nitric oxide synthase inhibitor $\mathrm{N}-(\mathrm{G})$-nitro-L-arginine methyl ester $\left(10^{-4} \mathrm{~mol} / \mathrm{l}\right.$; Cayman Chemical), the general cyclooxygenase inhibitor indomethacin (Indo, $10^{-5} \mathrm{~mol} / \mathrm{l}$; Cayman Chemical), PGE2 $\left(10^{-6} \mathrm{~mol} / \mathrm{l}\right.$; Cayman Chemical), and an antagonist specific for the EP4 subtype of PGE receptor, L-161982 ( $10^{-6} \mathrm{~mol} / \mathrm{l}$; Cayman Chemical). Drugs were dissolved in Krebs buffer or ethanol. Final solvent concentration in the bath was $\leq 0.04 \%$. In some experiments, vessels were preincubated with enzyme inhibitors or receptor antagonists for $60 \mathrm{~min}$ before isoprostane exposure. Vessels were exposed to each agent until a stable baseline diameter was reached (typically 20-30 min). Drug concentrations refer to their final molar concentration in the bath.

\section{Measurement of 8-Isoprostanes in Lung Tissue}

Litters $(n=4)$ were divided into three groups at the time of delivery. Lung tissues from representative pups were collected, snap frozen, and stored at $-80^{\circ} \mathrm{C}$ from: (i) fetuses without respiration $(n=8)$, (ii) pups exposed to room air for $4 \mathrm{~h}(n=8)$, and (iii) pups exposed to $80 \%$ oxygen for $4 \mathrm{~h}(n=8)$. Isoprostane content was determined on pooled lung tissues using well-established stable isotope dilution gas chromatography/negative-ion chemical ionization mass spectrometry (GC-NICI-MS) techniques as previously described (6). Levels of bound 8 -iso-PGF2a were indexed to sample weight in each group.

\section{Gene Expression Studies}

Total RNA obtained from pooled DA samples $(n=10-12$ DA segments per litter; $3-5$ representative litters/time point) underwent oligo(dT)-primed reverse transcription. Expression of the Tbxa2r gene, encoding the TP receptor for TxA2, and the Ptger4 gene, encoding the EP4 receptor for PGE2, was analyzed by realtime quantitative PCR (Roche Diagnostics, Indianapolis, IN). The sequences for oligonucleotide PCR primers included: TP-forward: 5'-GGTGGAGATGATGGTTCAGC, TP-reverse: 5'-TAGTCCACC AAGCACTCAGG,EP4-forward:5'-TCATCTTACTCATCGCCACC, EP4-reverse: 5'-GCACAGTCTTCCGAAGAAGG. Results were normalized to the expression of the housekeeping gene ribosomal protein L7 ( $r p l 7)$ (35).

\section{Statistical Analysis}

Gene expression and GC-NICI-MS studies were analyzed by ANOVA and Bonferroni post hoc analysis. For clarity, graph values are expressed as mean \pm SEM. Myography results were plotted as cumulative percent change from resting baseline or as percent reversal of preconstricted baseline. Independent lumen diameters were compared using ANOVA, and dependent diameters were compared using a paired $t$-test. Myography data were analyzed using a linear mixed-effects regression model controlling for baseline vessel diameter. A random intercept was included in each mixed-effects model to account for the correlation arising from measuring the diameter of the same vessel at multiple concentrations. Regression models were fitted using R (R Foundation, Vienna, Austria) and Stata, version 9 (Stata, College Station, TX) statistics software. $P<0.05$ was considered significant.

\section{ACKNOWLEDGMENTS}

We gratefully acknowledge the critical support and participation of Dr Jason D. Morrow (deceased) in these studies.

\section{STATEMENT OF FINANCIAL SUPPORT}

This work was supported by National Institutes of Health grants HL102042 (to J.-X. C.), HL075511 (to J.L.A.), and HL77395 and HL96967 (to J.R.).

Disclosure: The authors declare no conflict of interest.

\section{REFERENCES}

1. Hoffman JI, Kaplan S. The incidence of congenital heart disease. J Am Coll Cardiol 2002;39:1890-900.

2. Franco MC, Kawamoto EM, Gorjão R, et al. Biomarkers of oxidative stress and antioxidant status in children born small for gestational age: evidence of lipid peroxidation. Pediatr Res 2007;62:204-8.

3. Signorini C, Ciccoli L, Leoncini S, et al. Free iron, total F-isoprostanes and total F-neuroprostanes in a model of neonatal hypoxic-ischemic encephalopathy: neuroprotective effect of melatonin. J Pineal Res 2009;46:148-54.

4. Peter Stein T, Scholl TO, Schluter MD, et al. Oxidative stress early in pregnancy and pregnancy outcome. Free Radic Res 2008;42:841-8.

5. Buonocore G, Perrone S, Longini M, et al. Oxidative stress in preterm neonates at birth and on the seventh day of life. Pediatr Res 2002;52:46-9.

6. Milne GL, Yin H, Brooks JD, Sanchez S, Roberts LJ, Morrow JD. Quantification of F2-isoprostanes in biological fluids and tissues as a measure of oxidant stress. Meth Enzymol 2007;433:113-26.

7. Comporti M, Signorini C, Leoncini S, Buonocore G, Rossi V, Ciccoli L. Plasma F2-isoprostanes are elevated in newborns and inversely correlated to gestational age. Free Radic Biol Med 2004;37:724-32.

8. Rogers MS, Wang CC, Lau TK, et al. Relationship between isoprostane concentrations, metabolic acidosis, and morbid neonatal outcome. Clin Chem 2005;51:1271-4. 
9. Weinberger B, Nisar S, Anwar M, Ostfeld B, Hegyi T. Lipid peroxidation in cord blood and neonatal outcome. Pediatr Int 2006;48:479-83.

10. Cracowski JL, Durand T. Cardiovascular pharmacology and physiology of the isoprostanes. Fundam Clin Pharmacol 2006;20:417-27.

11. Janssen LJ. Isoprostanes: an overview and putative roles in pulmonary pathophysiology. Am J Physiol Lung Cell Mol Physiol 2001;280:L1067-82.

12. Belik J, González-Luis GE, Perez-Vizcaino F, Villamor E. Isoprostanes in fetal and neonatal health and disease. Free Radic Biol Med 2010; 48:177-88.

13. van der Sterren S, Villamor E. Contractile effects of 15-E2t-isoprostane and 15-F2t-isoprostane on chicken embryo ductus arteriosus. Comp Biochem Physiol, Part A Mol Integr Physiol 2011;159:436-44.

14. Belik J, Jankov RP, Pan J, Yi M, Chaudhry I, Tanswell AK. Chronic O2 exposure in the newborn rat results in decreased pulmonary arterial nitric oxide release and altered smooth muscle response to isoprostane. J Appl Physiol 2004;96:725-30.

15. Jankov RP, Luo X, Cabacungan J, et al. Endothelin-1 and O2-mediated pulmonary hypertension in neonatal rats: a role for products of lipid peroxidation. Pediatr Res 2000;48:289-98.

16. Luo X, Sedlackova L, Belcastro R, Cabacungan J, Lye SJ, Tanswell AK. Effect of the 21-aminosteroid U74389G on oxygen-induced free radical production, lipid peroxidation, and inhibition of lung growth in neonatal rats. Pediatr Res 1999;46:215-23.

17. Clarke DL, Belvisi MG, Hardaker E, Newton R, Giembycz MA. E-ring 8-isoprostanes are agonists at EP2- and EP4-prostanoid receptors on human airway smooth muscle cells and regulate the release of colonystimulating factors by activating cAMP-dependent protein kinase. Mol Pharmacol 2005;67:383-93.

18. Janssen LJ, Tazzeo T. Involvement of TP and EP3 receptors in vasoconstrictor responses to isoprostanes in pulmonary vasculature. J Pharmacol Exp Ther 2002;301:1060-6.

19. Crankshaw DJ, Rangachari PK. Isoprostanes: more than just mere markers. Mol Cell Biochem 2003;253:125-30.

20. Minuz P, Andrioli G, Degan M, et al. The F2-isoprostane 8-epiprostaglandin F2alpha increases platelet adhesion and reduces the antiadhesive and antiaggregatory effects of NO. Arterioscler Thromb Vasc Biol 1998;18:1248-56.

21. Fontana L, Giagulli C, Minuz P, Lechi A, Laudanna C. 8-Iso-PGF2 alpha induces beta 2 -integrin-mediated rapid adhesion of human polymorphonuclear neutrophils: a link between oxidative stress and ischemia/ reperfusion injury. Arterioscler Thromb Vasc Biol 2001;21:55-60.

22. Waleh N, Seidner S, McCurnin D, et al. Anatomic closure of the premature patent ductus arteriosus: The role of CD14+/CD163+ mononuclear cells and VEGF in neointimal mound formation. Pediatr Res 2011;70:332-8.

23. Echtler K, Stark K, Lorenz M, et al. Platelets contribute to postnatal occlusion of the ductus arteriosus. Nat Med 2010;16:75-82.

24. Rogers MS, Mongelli JM, Tsang KH, Wang CC, Law KP. Lipid peroxidation in cord blood at birth: the effect of labour. Br J Obstet Gynaecol 1998;105:739-44.

25. Friel JK, Friesen RW, Harding SV, Roberts LJ. Evidence of oxidative stress in full-term healthy infants. Pediatr Res 2004;56:878-82.

26. Signorini C, Perrone S, Sgherri C, et al. Plasma esterified F2-isoprostanes and oxidative stress in newborns: role of nonprotein-bound iron. Pediatr Res 2008;63:287-91.

27. Ahola T, Fellman V, Kjellmer I, Raivio KO, Lapatto R. Plasma 8-isoprostane is increased in preterm infants who develop bronchopulmonary dysplasia or periventricular leukomalacia. Pediatr Res 2004;56:88-93.

28. Longini M, Perrone S, Vezzosi P, et al. Isoprostane levels in urine of preterm newborns treated with ibuprofen for patent ductus arteriosus closure. Pediatr Nephrol 2011;26:105-9.
29. Reeve HL, Tolarova S, Nelson DP, Archer S, Weir EK. Redox control of oxygen sensing in the rabbit ductus arteriosus. J Physiol (Lond) 2001;533(Pt 1):253-61.

30. Michelakis ED, Rebeyka I, Wu X, et al. O2 sensing in the human ductus arteriosus: regulation of voltage-gated $\mathrm{K}+$ channels in smooth muscle cells by a mitochondrial redox sensor. Circ Res 2002;91:478-86.

31. Thébaud B, Michelakis ED, Wu XC, et al. Oxygen-sensitive Kv channel gene transfer confers oxygen responsiveness to preterm rabbit and remodeled human ductus arteriosus: implications for infants with patent ductus arteriosus. Circulation 2004;110:1372-9.

32. Kajimoto H, Hashimoto K, Bonnet SN, et al. Oxygen activates the Rho/ Rho-kinase pathway and induces RhoB and ROCK-1 expression in human and rabbit ductus arteriosus by increasing mitochondria-derived reactive oxygen species: a newly recognized mechanism for sustaining ductal constriction. Circulation 2007;115:1777-88.

33. Sametz W, Hennerbichler S, Glaser S, Wintersteiger R, Juan H. Characterization of prostanoid receptors mediating actions of the isoprostanes, 8-iso-PGE(2) and 8-iso-PGF(2alpha), in some isolated smooth muscle preparations. Br J Pharmacol 2000;130:1903-10.

34. McNamara P, Lawson JA, Rokach J, FitzGerald GA. Isoprostane activation of the nuclear hormone receptor PPAR. Adv Exp Med Biol 2002;507:351-5.

35. Reese J, Paria BC, Brown N, Zhao X, Morrow JD, Dey SK. Coordinated regulation of fetal and maternal prostaglandins directs successful birth and postnatal adaptation in the mouse. Proc Natl Acad Sci USA 2000;97:9759-64.

36. Loftin CD, Trivedi DB, Tiano HF, et al. Failure of ductus arteriosus closure and remodeling in neonatal mice deficient in cyclooxygenase- 1 and cyclooxygenase-2. Proc Natl Acad Sci USA 2001;98:1059-64.

37. Nguyen M, Camenisch T, Snouwaert JN, et al. The prostaglandin receptor EP4 triggers remodelling of the cardiovascular system at birth. Nature 1997;390:78-81.

38. Reese J, Anderson JD, Brown N, Roman C, Clyman RI. Inhibition of cyclooxygenase isoforms in late- but not midgestation decreases contractility of the ductus arteriosus and prevents postnatal closure in mice. Am J Physiol Regul Integr Comp Physiol 2006;291:R1717-23.

39. Reese J, O'Mara PW, Poole SD, et al. Regulation of the fetal mouse ductus arteriosus is dependent on interaction of nitric oxide and COX enzymes in the ductal wall. Prostaglandins Other Lipid Mediat 2009;88:89-96.

40. Leonhardt A, Glaser A, Wegmann M, Schranz D, Seyberth H, Nüsing R. Expression of prostanoid receptors in human ductus arteriosus. Br J Pharmacol 2003;138:655-9.

41. Rheinlaender C, Weber SC, Sarioglu N, Strauss E, Obladen M, Koehne P. Changing expression of cyclooxygenases and prostaglandin receptor EP4 during development of the human ductus arteriosus. Pediatr Res 2006;60:270-5.

42. Trivedi DB, Sugimoto Y, Loftin CD. Attenuated cyclooxygenase-2 expression contributes to patent ductus arteriosus in preterm mice. Pediatr Res 2006;60:669-74.

43. Jourdan KB, Evans TW, Curzen NP, Mitchell JA. Evidence for a dilator function of 8-iso prostaglandin $\mathrm{F} 2$ alpha in rat pulmonary artery. $\mathrm{Br} \mathrm{J}$ Pharmacol 1997;120:1280-5.

44. Richard C, Gao J, LaFleur B, et al. Patency of the preterm fetal ductus arteriosus is regulated by endothelial nitric oxide synthase and is independent of vasa vasorum in the mouse. Am J Physiol Regul Integr Comp Physiol 2004;287:R652-60.

45. Dröge W. Free radicals in the physiological control of cell function. Physiol Rev 2002;82:47-95. 